prevention, support for junior nursing staff and the development of the non-registered workforce.

Increasing the support for lower dependency care can potentially relieve pressures upstream. Solutions for workforce transformation cannot be considered in isolation. It has become apparent that organisation and service delivery of care has to be considered in tandem for workforce transformation.

Results To further explore:

- Nursing Associates

- Role definition for Enhanced Neonatal Nurse Practitioners

- Development of Non-Medical Supporting Roles

- Development of Associate Specialist/MTIp roles

- Awareness of impact of culture and morale on the workforce

- Investment in clinical supervision, education and training

- Development of networked preceptorship nursing programmes

- Development of Transitional Care

- Implementation of ATAIN programme

- Implementation of Integrated Family Development Care

However, despite all best intentions, these roles and service developments may not have a sustainable effect on vacancies in middle grade doctors or nurses achieving QIS. It is important to recognise this as a limitation.

\section{G202(P) OUTCOMES OF EXTREMELY LOW BIRTH WEIGHT INFANTS - THE EFFECT OF BEING SMALL FOR GESTATIONAL AGE}

${ }^{1} \mathrm{E}$ Charles, ${ }^{1} \mathrm{C}$ Harris, ${ }^{2} \mathrm{~A}$ Hickey, ${ }^{1} \mathrm{~A}$ Greenough. ${ }^{1}$ Women and Children's Health, School of Life Course Sciences, Faculty of Life Sciences and Medicine, King's College London, London, UK; ${ }^{2}$ Neonatal Intensive Care Centre, King's College Hospital NHS Foundation Trust, London, UK

\subsection{6/archdischild-2018-rcpch.197}

Aims Prematurely-born infants who are small for gestational age (SGA) have worse outcomes than those who are born appropriate for gestational age (AGA). ${ }^{1,2}$ There has, however, been little focus on SGA infants born at extremely low birth weights (BW <750 g).

Methods A retrospective study was undertaken of all babies BW <750 gm born between 2012 and 2016. Centiles and zscores were calculated using the UK WHO preterm reference ranges (British 1990 reference data, reanalysed 2009). The infants were divided into SGA $(<10$ th centile) and AGA (10th -90 th centile) groups. Multiple logistic regression analysis was performed to adjust for gestational age to relate individual outcome variables to SGA.

Results Eighty-four infants were included, 35 (42\%) were SGA. The SGA infants were more mature (median gestational age 26.9 versus 24.3 weeks, $\mathrm{p}<0.001$ ), had a lower birth weight centile (median 1 versus 26, p<0.001); their mothers were more likely to have received antenatal steroids $(94 \%$ versus 78\%, $p=0.022)$, had hypertension $(49 \%$ versus $8 \%$, $\mathrm{p}<0.001)$ and be delivered by caesarean section $(69 \%$ versus $8 \%, \mathrm{p}<0.001)$. Neither the mortality $(31 \%$ versus $32 \%)$ nor the incidence of NEC (17\% versus 16\%) differed significantly between the two groups, but more of the SGA developed severe BPD $(p=0.025)$. The SGA infants achieved full enteral feeds at an older postnatal age (median 54 versus 48 days, $\mathrm{p}=0.019)$. The length of stay was similar in the two groups (127 versus 131 days), but the weight $\mathrm{z}$ score at discharge was lower in the SGA group $(-3.6$ versus -1.7$)(p=0.001)$. Indeed, there was no significant difference in the change in $\mathrm{z}$ score from birth between the two groups (median -1.53 versus $-1.07, \mathrm{p}=0.306)$.

Conclusions Amongst infants with a BW $<750$ gm, SGA compared to AGA suffered greater morbidity, but not mortality. These data are important for counselling parents as, in this population, any advantage of later gestation may be negated by being SGA.

\section{REFERENCES}

1. Peacok JL, et al. Pediatr Res 2013;73:457-63.

2. Tsai L-Y, et al. Pediatr Neonatol 2015;56:101-7.

\section{G203(P) UK NEONATAL RESUSCITATION SURVEY}

E Charles, K Hunt, A Milner, A Greenough. Women and Children's Health, School of Life Course Sciences, Faculty of Life Sciences and Medicine, King's College London, London, UK

\subsection{6/archdischild-2018-rcpch.198}

Aim In the UK, neonatal resuscitation practice follows national guidelines set by the UK Resuscitation Council. Two previous surveys, however, found significant differences in practice between units according to the level of care offered. ${ }^{1,2}$ Since then changes have been made to the guidelines ${ }^{3}$ and there has been interest in the role of carbon dioxide (CO2) monitoring during neonatal resuscitation. ${ }^{4}$ Our aim was to determine if these changes had altered resuscitation practice in the delivery suite across the UK.

Methods An online questionnaire was sent to the lead consultants of 189 units. If no response was received, a follow up email was sent and further non-response was followed by a telephone call. The results were compared to the 2012 survey. ${ }^{1}$

Results Over all, there was an $83 \%$ response rate per level of unit: (neonatal intensive care unit (NICU) 93\%, local neonatal unit (LNU) 83\%, special care baby unit (SCBU). Currently all units used an initial inspired oxygen ( $\mathrm{FiO} 2)$ of 0.21 for term born infants, whereas previously it had been used in $84.5 \%$ NICUs, $39.5 \%$ of LNUs and $31.7 \%$ of SCBUs. An initial FiO2 of 0.21 for preterm infants was used in NICUs, LNUs and SCBUs in 2017 in $86 \%, 93 \%$ and 93\% respectively and in 2012 in $42 \%, 22 \%$ and $24 \%$ respectively. Routine use of oxygen saturation monitoring for preterm infants had increased from $71 \%, 65 \%$ and $42 \%$ in 2012 to $94 \%, 88 \%$ and $84 \%$ (NICUs, LNUs and SCBUs respectively). CO2 monitoring was also more common, currently used by $88 \%, 81 \%$ and $84 \%$ compared to $24 \%-20 \%$ and $10 \%$ in 2012 (NICUs, LNUs and SCBUs respectively).

Conclusion There have been marked changes in delivery suite resuscitation practices since 2012 and more consistency across levels on units.

\section{REFERENCES}

1. Murthy V, et al. Arch Dis Child Fetal Neonatal Ed 2012;97:F154-5.

2. Mann C, et al. Resuscitation 2012;83:607-11.

3. Wyllie J, et al. European Resuscitation Council Guidelines for Resuscitation 2015.

4. Hawkes G, et al. Resuscitation 2014;85:1315-9. 